\title{
Route maintenance and Scalability improvement of DSR, based on Relay node identification after locating Link-failure over MANET
}

\author{
Sourish Mitra ${ }^{1} \underset{1,2,3,4,5}{\text { Mounita } \text { Das }^{2}, \text { Rupa Mitra }}{ }^{3}$, Priyanka Maity ${ }^{4}$, Alolika Banerjee ${ }^{5}$ \\ (Department of Computer Science \& Engineering, Gurunanak Institute of Technology, India)
}

\begin{abstract}
In Dynamic Source Routing, each source determines the route to be used in transmitting its packets to destination. Route Discovery determines the optimum path for a transmission between a given source and destination. Route Maintenance ensures that the transmission path remains optimum and loop-free as network conditions change, even if this requires changing the route during a transmission. Many protocols were proposed to maintain route from high data loss and transition delay due to frequent link failure but doesn't take the decision on basis of location of failure link in source route and also doesn't take advantages of Relay node identification in partitioned source route. From this point of view in our paper we propose an algorithm with the help of local link repairing and improvement strategy based on the location of link failure inside source route over mobile ad-hoc network. Through this paper we introduce our proposed approach based on DSR, which takes decision on the basis of the location of Relay node(where link failure is detected) in source route. We think our new approach can be able to improve packet salvaging, packet delivery ratio and reducing end to end delay to improve the scalability of Mobile Ad-hoc network.
\end{abstract}

Keywords: End to end delay, frequent link failure, packet delivery ratio, packet salvaging, relay node.

\section{Introduction}

In the routing of mobile ad hoc networks (MANET), frequent link breaks occur in the path due to unrestricted node mobility and node failures which violate the quality of service $(\mathrm{QoS})$ requirements. Routing in MANET is critical due to its dynamic topology. In Case of Reactive routing protocol every node in the network obtains a route to a destination on a demand fashion. Reactive protocols do not maintain up-to-date routes to any destination in the network and do not generally exchange any periodic control messages. Dynamic Source Routing (DSR) is a reactive routing protocol for wireless mesh networks. It is similar to AODV in that it forms a route on-demand when a transmitting computer requests one. However, it uses source routing instead of relying on the routing table at each intermediate device. This protocol is truly based on source routing whereby all the routing information is maintained (continually updated) at mobile nodes. It has only two major phases, which are Route Discovery and Route Maintenance. Route Reply would only be generated if the message has reached the intended destination node (route record which is initially contained in Route Request would be inserted into the Route Reply).In case of route discovery the source sends a broadcast packet which contains source address, destination address, request id and path. If a host saw the packet before, discards it. Otherwise, the route looks up its route caches to look for a route to destination, If not find, appends its address into the packet rebroadcast if finds a route in its route cache, sends a route reply packet, which is sent to the source by route cache or the route discovery. On other hands Route Maintenance indicates whenever a node transmits a data packet, a route reply, or a route error, it must verify that the next hop correctly receives the packet. If not, the node must send a route error to the node responsible for generating this route header intermediate nodes "eavesdrop", adjust cached routes. Source deletes route; tries another if one cached, or the source restart the route discovery.

\section{Link Failure In Manet}

On-demand routing protocol relies on global flooding of route request packet for the route discovery. Once the source performs the route discovery successfully, data are sent via the discovered route. However, due to the dynamic and mobile nature of the nodes, intermediate nodes in the route tend to lose connection with each other during the communication process, that is, the route is likely to be broken. When this occurs, different protocols may take different actions to re-establish a route in order to maintain the communication channel between the source and destination nodes.

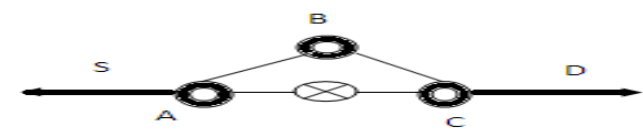

fig. Link failure in Manet 
According to above figure (Section-II), a route is discovered and established between the source node $\mathrm{S}$ and the destination node D. Nodes A and B are intermediate nodes in the established route. In the situation that the link between A and its next node B break off, the route between S and D is said to be broken. When DSR is used, A sends back an ERROR message to the data source $S$ after which $S$ initiates another round of route discovery process in an attempt to find a new route to D. This mechanism, used by DSR, is also known as end-to-end route recovery and is not scalable.

\section{Assumption Of Local Link Repairing Mechanism In Manet}

According to above Figure, if the direct link $(\mathrm{A} \rightarrow \mathrm{B})$ breaks off, there should exist, in most cases, some indirect route from $\mathrm{A}$ to the original next node $\mathrm{B}$ through some neighbour node $\mathrm{C}$. In these situations, if a request packet is sent out to find the original next hop or other node which is at the further part of the original route with limited time-to-live (eg. 2 hops), the possibility of repairing the current route should be high and the overhead should be much lower than using end-to-end global recovery.

\section{Local Link Repairing Process In Manet}

According to above figure (Section-II) and also with above assumptions, an algorithm was proposed which described below: When an intermediate node A discovers that the link to the next hop has broken, it would:

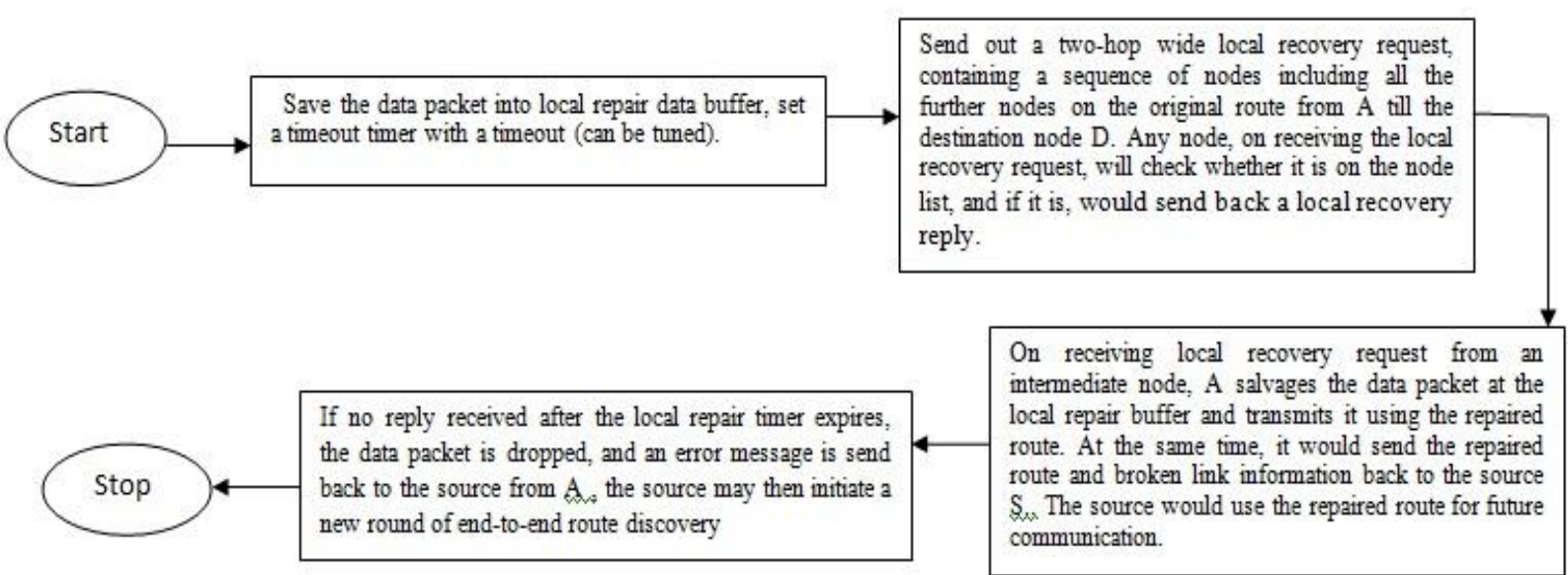

Fig. Local Link Repairing Mechanism

V. Route Repairing With Down Stream Node Information In Manet

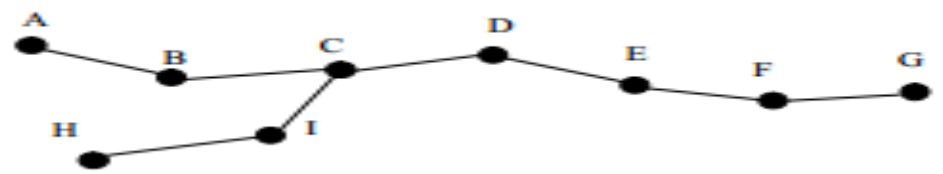

Fig. Down Stream Node Information in Manet

Consider two paths, $\mathrm{P}(\mathrm{A}, \mathrm{B}, \mathrm{C}, \mathrm{D}, \mathrm{E}, \mathrm{F}, \mathrm{G})$ and $\mathrm{P}(\mathrm{H}, \mathrm{I}, \mathrm{C}, \mathrm{D}, \mathrm{E}, \mathrm{F}, \mathrm{G})$, in the network as shown in above Figure. Suppose when node $C$ sends a data packet to node $G$ along $P(A, B, C, D, E, F, G), C$ found out that the link $(\mathrm{E}, \mathrm{F})$ is broken. Then, this information is disseminated to all upstream nodes of $\mathrm{C}$ in both paths. On receiving the link breakage information nodes $A, B, H$, and I modify the paths by removing sub path $P(F, G)$. Otherwise, attempting to use this broken link may cause delay. At current node $\mathrm{C}$ when an existed broken link is found between node $\mathrm{E}$ and $\mathrm{F}$ then for each path $\mathrm{P}$ in the Routing Table that contains a link (E, F). So we can write, $\mathrm{P}$ - rem $(\mathrm{P}, \mathrm{F})$. Sends a Broken Link Message to its upstream nodes, if any, of all paths that contain (E, $\mathrm{F})$. if $\mathrm{C}$ received a broken link message from its downstream node in an existing path, then for each path $\mathrm{P}$ in the Routing Table that contains a link (E, F) . So, P - rem (P, F) Sends a Broken Link Message to its upstream nodes, if any, of all paths that contain $(\mathrm{E}, \mathrm{F})$. 


\section{Our Proposed Algorithm}

Many Route repair mechanisms for MANET are suffering from certain limitations. The main limitation of DSR is more bandwidth consumption due to flooding. Lots of error messages makes congestion in ad-hoc network and also more packet drops creates huge difficulties. Due to Local Link Recovery Mechanisms Network always takes decision on the basis of local information and overhead on all intermediate nodes are more and all intermediate node work on same mechanism irrespective of their location in the source route. This is really a limitation. Even in case of Downstream Nodes Information if failed link is far away from the destination then it may cause overhead on intermediate nodes and if nodes are highly movable then more links failure occurs and may degrade the performance of the network. In our proposed algorithm we think that we can overcome all of these aforesaid limitations on Mobile Ad-Hoc Network. Consecutive steps of our proposed approach are given below.

Step 1: Relay node first makes a search it's route information buffer and find out that is there any route attached with destination or not.

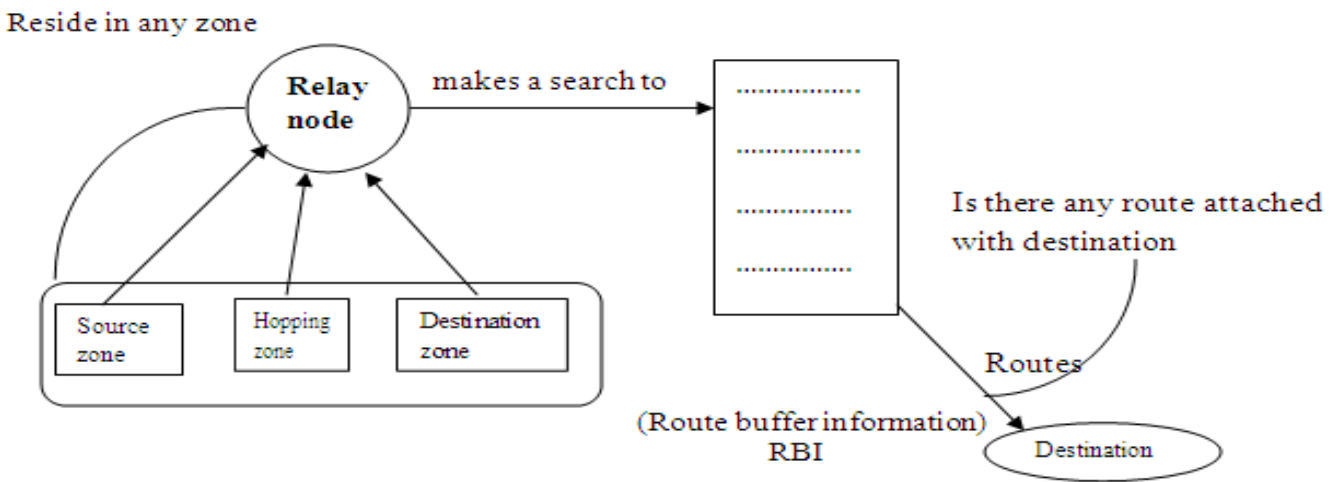

Step 2: If there is no link failure and if route found then forward the packet through that informed route to destination and inform to source about this route.

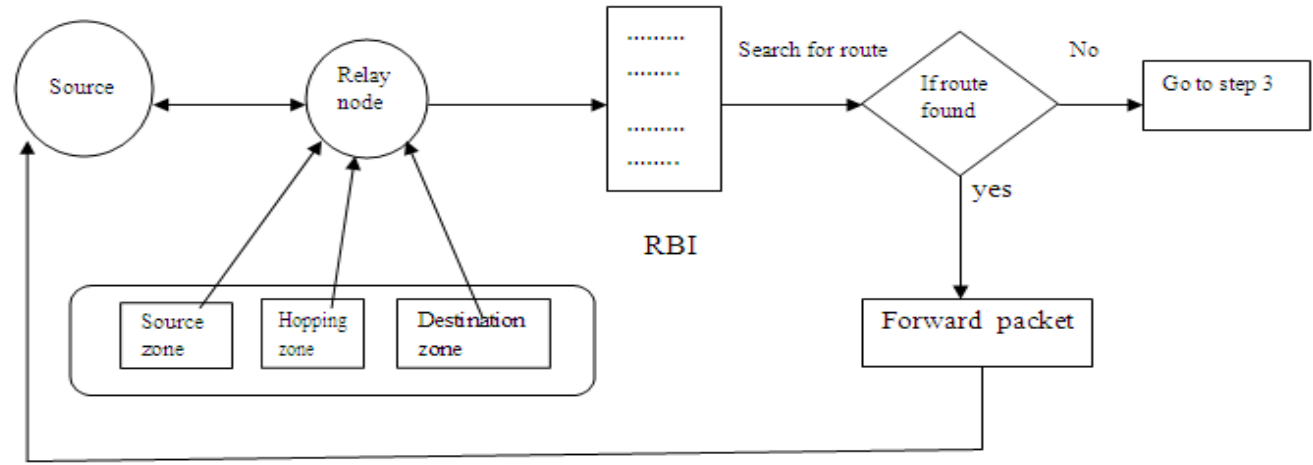

Step 3: If route does not found then relay node identifies its location in the failed source route.

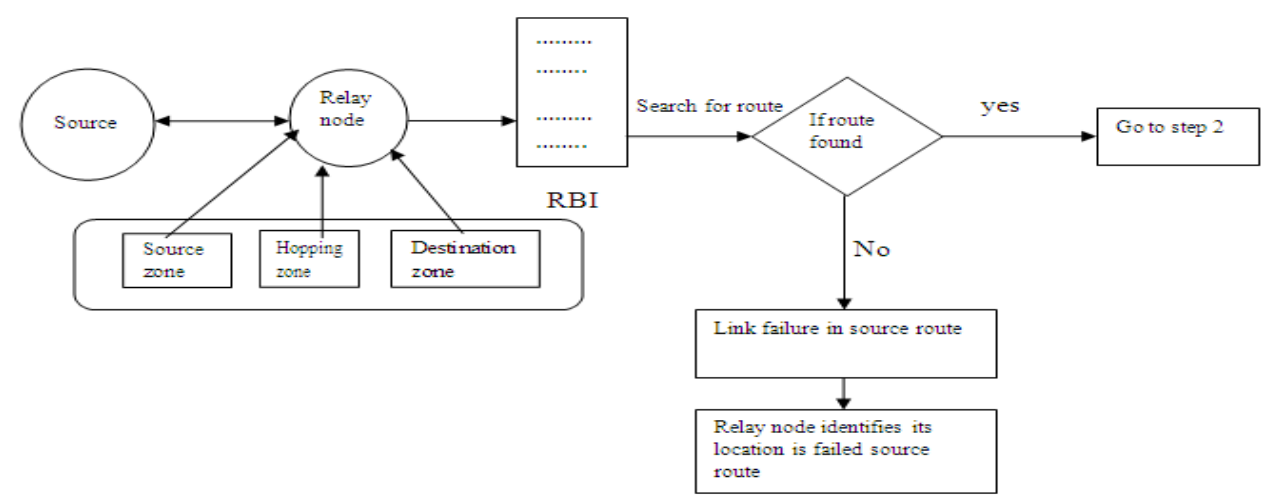


Step 4: Relay node can be identified at any position in the source route over MANET. So we can introduce our proposed concept i.e zone partitioning of source route and localized existence of relay node inside on it. Here three cases arise.

Case 1: Existence of relay node inside source within its first neighbour zone over the source route.

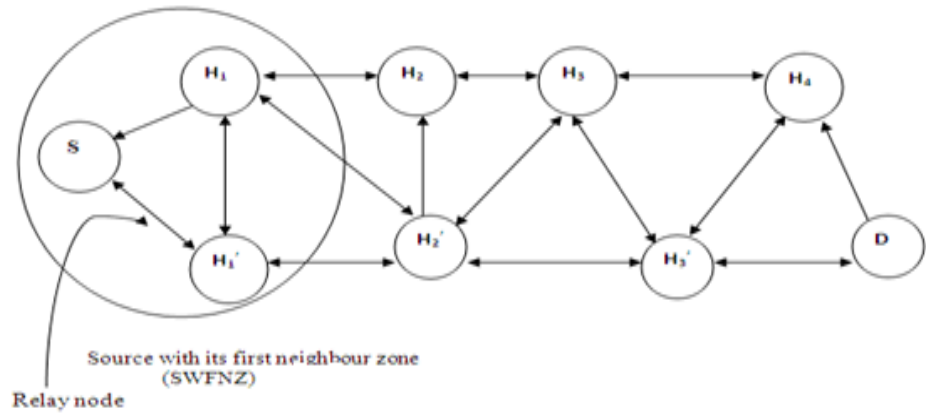

Fig. Relay node inside source with its first neighbour zone

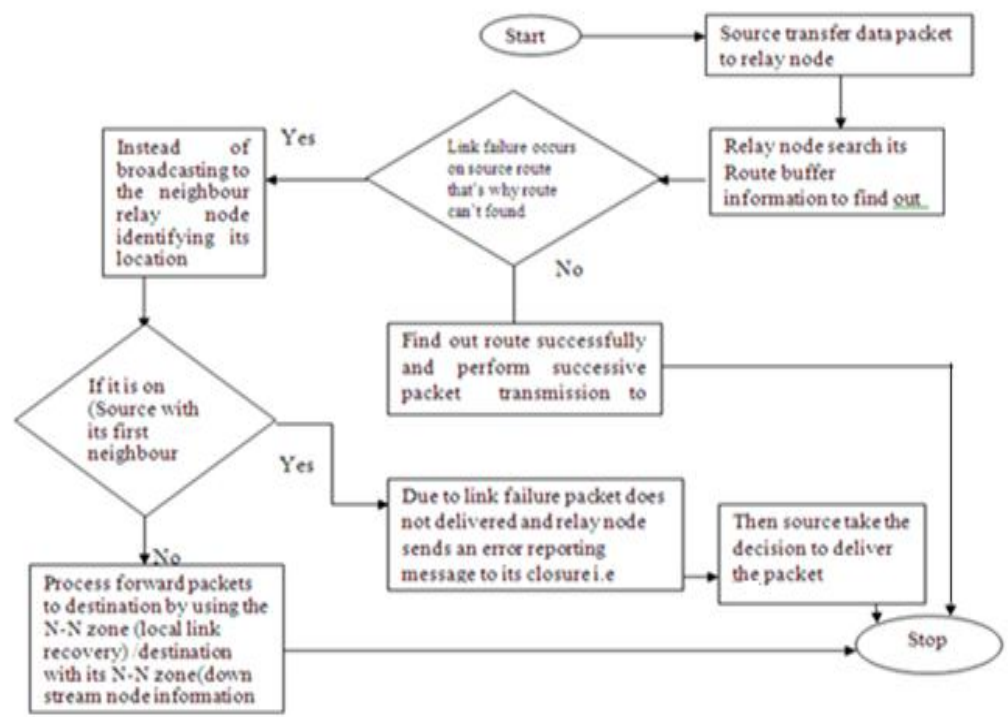

Fig. Proposed flow chart with activities when realy node is on source with its first neighbour zone

Case 2: Existence of relay node inside neighbour to neighbour $(\mathrm{N}-\mathrm{N})$ zone over the source route.

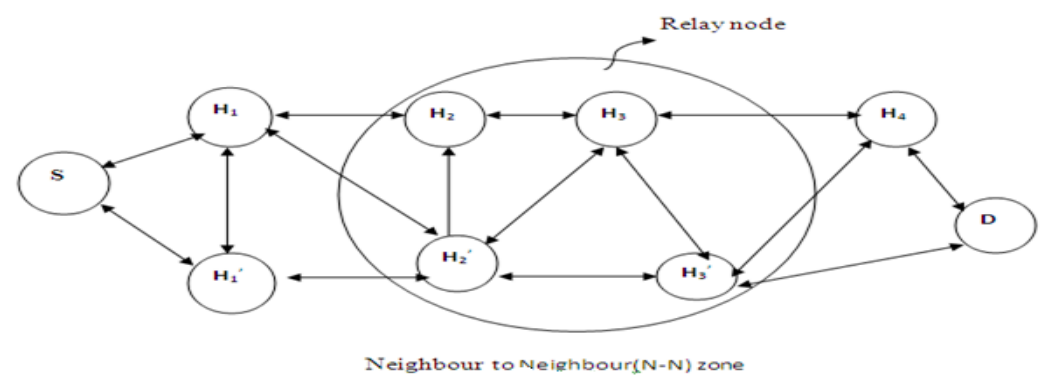

Fig. Relay node inside neighbour to neighbour zone 


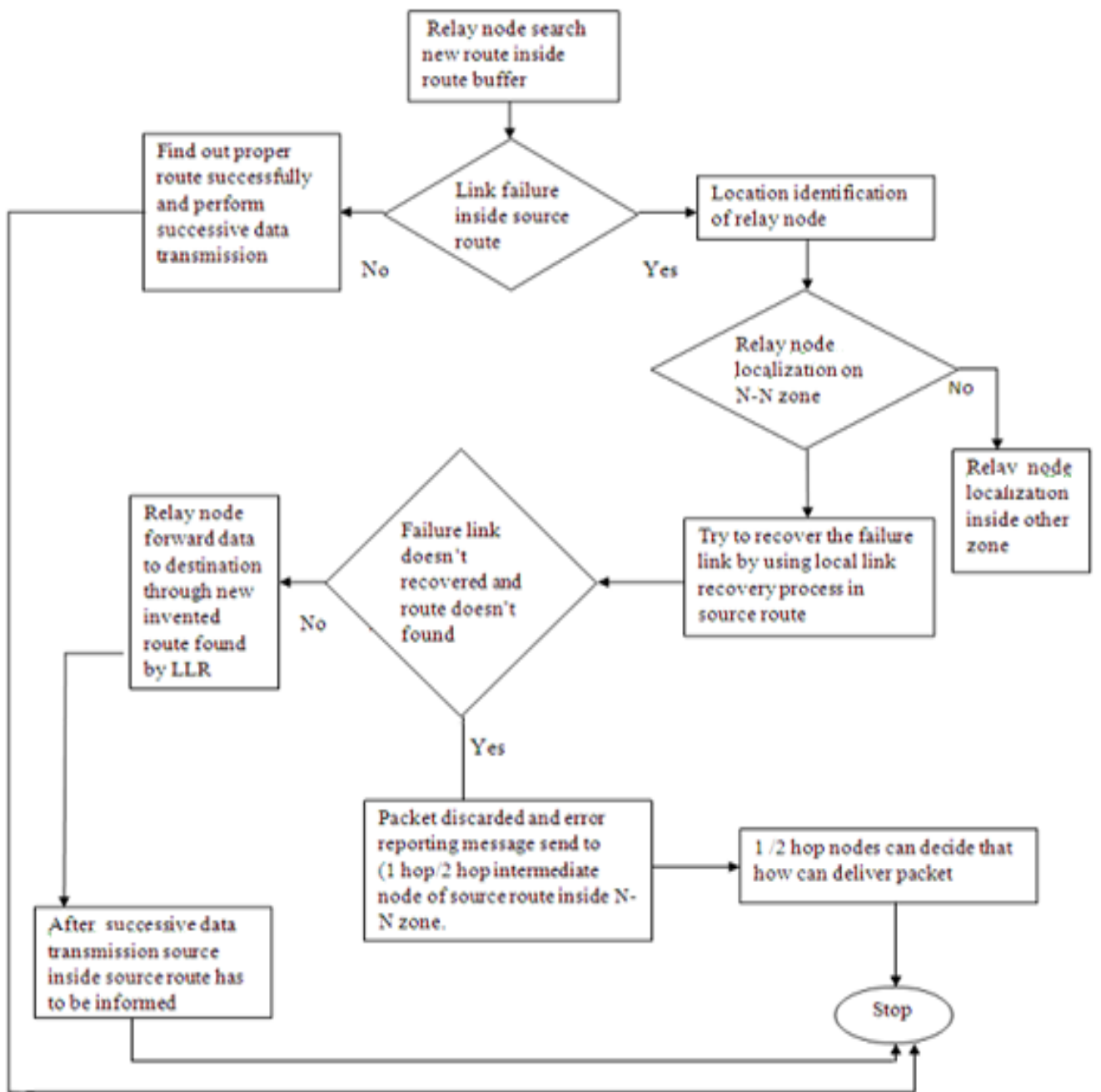

Fig.Proposed flow chart with activities when relay node is on neighbour to neighbour zone

Case 3: Existence of relay node inside Destination with its nearest neighbour zone over the source route.

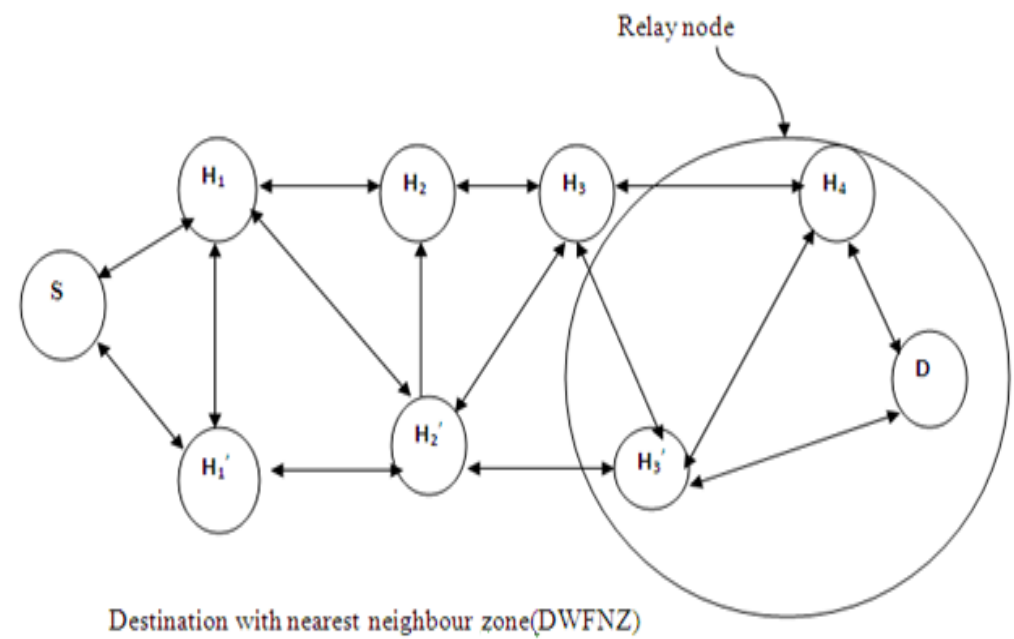

Fig. Relay node inside destination with nearest neighbour zone 


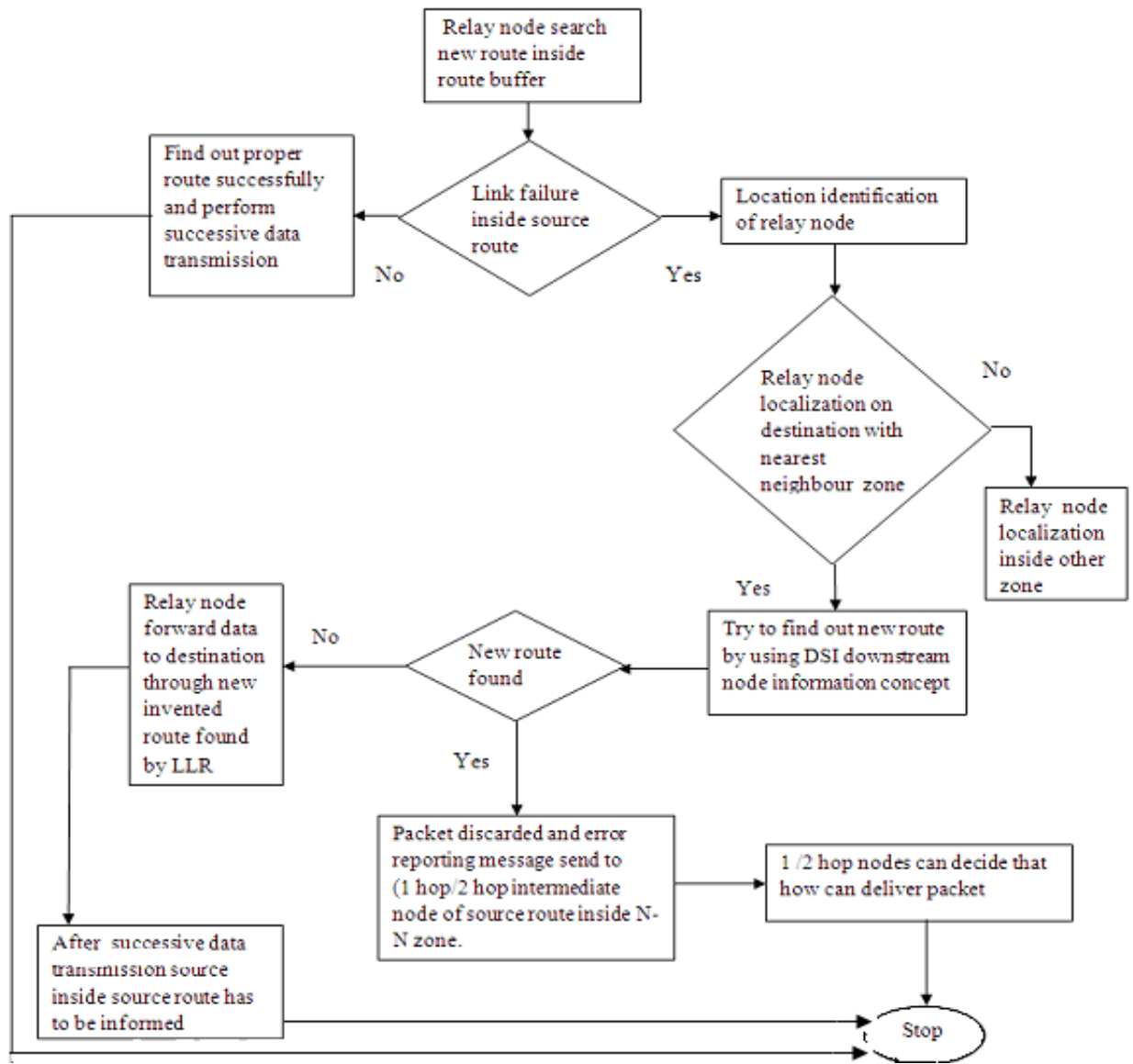

Fig.Proposed flow chart with activities when relay node is on distance with nearest neighbour zone

\section{Result Of Experiment With Simulation}

We can use NS-2 simulator to evaluate our proposed approach. We compare our approach with DSR approach. Following graph shows the result analysis which can be achieved by NS-2.

Packet delivery ratio: The ratio of the number of delivered data packet to the destination. This illustrates the level of delivered data to the destination.

\section{$\sum$ Number of packet receive $/ \sum$ Number of packet send}

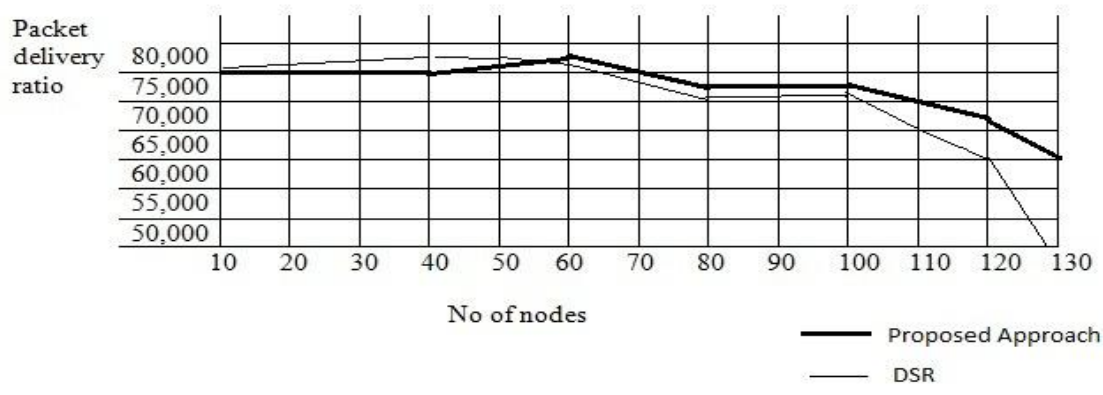

Fig1. PDR in our proposed algorithm vs PDR in DSR

End to End delay: The average time taken by a data packet to arrive in the destination. It also includes the delay caused by route discovery process and the queue in data packet transmission. Only the data packets that successfully delivered to destinations that counted.

\section{$\sum$ (arrive time - send time) $/ \sum$ Number of connections}




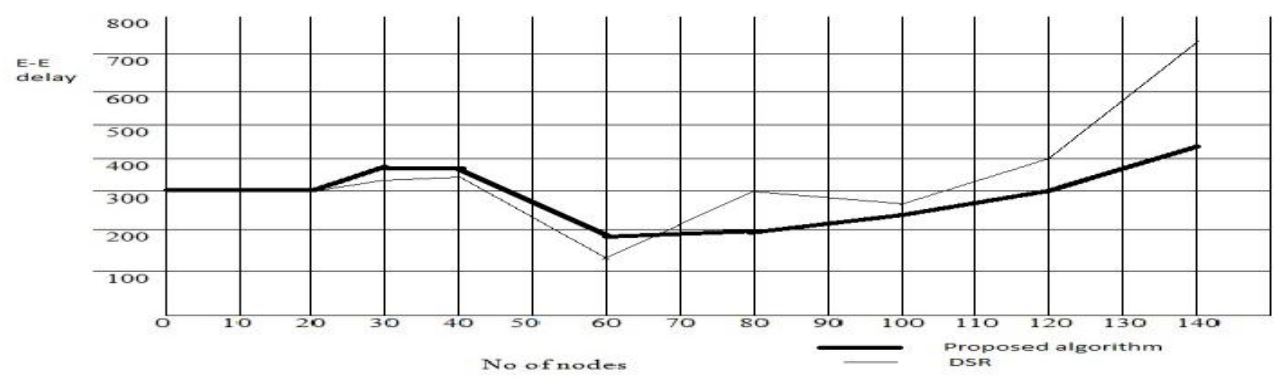

Fig2. E-E delay: Proposed Algorithm vs DSR

Through Fig1, we can see that PDR $\infty$ no of nodes, but after a certain range no of nodes are inversely proportional to PDR. i.e according to the graph, when no of nodes $>60$ then PDR $\infty$ ( $1 /$ no of nodes).Through Fig2, we can see that if no of nodes is less then(E-E delay of DSR $\sim$ E-E delay of our approach)is very less. If no of nodes increases, then E-E delay of our approach $<<$ E-E delay of DSR.

\section{Conclusion}

Our new approach can be able to improve packet salvaging, packet delivery ratio and reducing end to end delay to improve the route maintenance as well as scalability of Mobile Ad-hoc network. According to our proposed route maintenance algorithm we can identify the location of Relay node when link failure occurs on source route. After identification inside a particular zone it will execute its proposed technique to deliver the data packet to the destination. Our approach also reduces no of error message inside the Ad-hoc network .Thus we can improve scalability of DSR. In our paper we propose an algorithm with the help of local link repairing and improvement strategy based on the location of link failure inside source route over mobile ad- hoc network. Through this paper we introduce our proposed approach based on DSR, which takes decision on the basis of the location of Relay node (where link failure is detected) in source route.

\section{References}

[1] “Implementation Of Dynamic Source Routing (DSR) In Mobile Ad Hoc Network (Manet)", Thiyam Romila Devi,, Rameswari Biswa, Vikram Kumar, Abhishek Jena,International Journal of Research in Engineering and Technology eISSN: 2319-1163 | pISSN: 2321-7308Volume: 02 Issue: 11 ,Nov-2013

[2] Genping Liu, Kai Juan Wong, Bu Sung Lee, Boon Chong Seet, Chuan Heng Foh, Lijuan Zhu, "PATCH: a novel local recovery mechanism for mobile ad-hoc networks", Vehicular Technology Conference, 2003. VTC 2003-Fall. 58th. Page(s):2995 - 2999 Vol.5. IEEE, 2003.

[3] Junjie Chen, Chang'en Zhou, Deli Chen, Bin Huang, Jiajun Hong, Chao Zhou1, Xiao Yang, "A Novel Routing Algorithm for Ad hoc Networks Based on the Downstream Nodes Information", International Conference on Multimedia information Networking and Security, 2009. 978-0-7695-3843-3/09 IEEE 2009.

[4] I.Vijaya , Amiya Kumar Rath, Pinak Bhusan Mishra, Amulya Ratna Dash, "Influence of Routing Protocols in Performance of Wireless Mobile Adhoc Network", Second International Conference on Emerging Applications of Information Technology. 978-07695-4329-1/11. DOI 10.1109/EAIT.2011.65. IEEE, 2011

[5] D.B. Johnson and D. A. Maltz, "Dynamic Source Routing Protocol for Mobile Ad Hoc Networks", Mobile Computing, T. Imielinski and H. Korth, Eds., Kluwer, 1996, pp. 153-81.

[6] C.E. Perkins and E. M. Royer, “Ad-hoc On-Demand Distance Vectored Routing”, Proc. 2nd IEEe Workshop. Mobile Comp. Sys. And Apps., Feb. 1999, pp. 90-100.

[7] Aron and S. Gupta. "A Witness-Aided Routing Protocol for Mobile Ad-Hoc Networks with Unidirectional Links", Proc. First lnt Conf. on Mobile Data Access (MDA '99), Hong-Kong, Dec. 1999, pp. 24-33.

[8] C-K. Toh, "A novel distributed routing protocol to support ad-hoc mobile computing," IEEE International Phoenix Conf. on Computers and Communications, IPCCC'96.

[9] C. K. Toh, "Long-lived ad hoc routing based on the concept of associativity", Internet draft, IETF, Mar. 1999.

[10] Tzay-Farn Shih, Hsu-Chun Yen, "Location aware routing protocol with dynamic adaptation of request zone for mobile ad hoc networks", Springer Wireless Network (2008) 14:321-333,DOI10.1007/s11276-006-9955-y

[11] International Journal of Computer applications (0975 - 888) Volume 47- No.4, June 2012. Reputation based Dynamic Source Routing Protocol for MANET 